\title{
Imported cutaneous leishmaniasis: molecular investigation unveils Leishmania major in Bangladesh
}

\author{
Md Anik Ashfaq Khan ${ }^{1}$, Rajashree Chowdhury ${ }^{1}$, Rupen Nath', Sören Hansen², Progga Nath ${ }^{3}$, Shomik Maruf ${ }^{1}$, \\ Ahmed Abd El Wahed ${ }^{2^{*}}$ (D) and Dinesh Mondal ${ }^{* *}$
}

\begin{abstract}
Background: The main clinical forms of leishmaniasis in Bangladesh are visceral leishmaniasis and post-kala-azar dermal leishmaniasis, which are caused by Leishmania donovani. Imported cutaneous leishmaniasis $(\mathrm{CL})$ is emerging globally due mainly to increased human mobility. In recent years, several imported $\mathrm{CL}$ cases have also been reported in Bangladesh. Sporadic atypical cases of $\mathrm{CL}$ can be challenging for diagnosis and clinical management, while occurrence of infection on a frequent basis can be alarming. We report of a case of a Bangladeshi temporary-migrant worker who, upon return, presented development of skin lesions that are characteristic of $\mathrm{CL}$.
\end{abstract}

Methods: A serum sample was collected and tested with an rK39 immunochromatographic test. Nucleic acid from skin biopsy derived culture sample was extracted and screened with a real-time PCR assay which targets the conserved REPL repeat region of $L$. donovani complex. The internal transcribed spacer 2 region of the ribosomal RNA gene cluster was amplified and sequenced.

Results: The suspect had a history of travel in both $C L$ and VL endemic areas and had a positive rK39 test result. Based on clinical presentation, travel history and demonstration of the parasite in the skin biopsy, CL was diagnosed and the patient underwent a combination therapy with Miltefosine and liposomal amphotericin B. While typical endemic species were not detected, we identified Leishmania major, a species that, to our knowledge, has never been reported in Bangladesh.

Conclusions: Proper monitoring and reporting of imported cases should be given careful consideration for both clinical and epidemiological reasons. Molecular tests should be performed in diagnosis to avoid dilemma, and identification of causative species should be prioritized.

Keywords: Cutaneous leishmaniasis, Bangladesh, Leishmania major, Imported infection, Sequencing

\section{Background}

Leishmaniasis is a group of insidious infectious diseases caused by species of the protozoan genus Leishmania transmitted through the bite of sand flies. It is classified into three clinical forms based upon the affected tissue, namely cutaneous (CL), mucocutaneous (MCL)

\footnotetext{
*Correspondence: abdelwahed@gwdg.de; din63d@icddrb.org

${ }^{1}$ Nutrition and Clinical Services Division, International Centre

for Diarrhoeal Disease Research, Bangladesh, Dhaka, Bangladesh

${ }^{2}$ Division of Microbiology and Animal Hygiene, Georg-August-Universität

Göttingen, Göttingen, Germany

Full list of author information is available at the end of the article
}

and visceral (VL) leishmaniasis. The disease is endemic in many parts of the world. Bangladesh belongs to the endemic zones for VL as well as its skin complication known as post-kala-azar dermal leishmaniasis (PKDL), both of which are caused by Leishmania donovani. A regional initiative for VL elimination, known as the regional kala-azar Elimination Programme (KAEP) have contributed to a remarkable decline in the incidence rate of VL cases over recent years in Bangladesh and other endemic regions of the Indian subcontinent; it is now approaching the maintenance phase of elimination [1]. Patients affected by CL or MCL, on the other hand, are 
not usually found in Bangladesh, which might be due to the absence of specific transmitting vectors [2]. The diagnostic and clinical practices are well-defined in the local health care centres for VL and PKDL, which is not the case for CL or MCL. Upon occurrence, the atypical disease forms may cause diagnostic and clinical dilemmas with respect to clinical presentation, cross-reaction in serological tests, and treatment strategies [3, 4]. Systematic investigation of atypical cases and identification of the causative Leishmania species are also important for epidemiological reasons. Here, we report of a temporarymigrant worker who was diagnosed with $\mathrm{CL}$ after his return to Bangladesh. We identified L. major as the causative agent. To our knowledge, this is the first report of a L. major infected CL case in Bangladesh.

\section{Methods}

\section{Case presentation}

A 40-year-old male was referred from the M.A.G Osmani Medical College Hospital, Sylhet to the Surya Kanta Kalaazar Research Center (SKKRC) hospital, Mymensingh in October 2017 as a suspected case of CL, with multiple skin lesions on his left forearm. However, no other anomaly such as fever, hepato-splenomegaly or mucosal lesion was observed. The patient had no history of VL, although he had been in to VL endemic areas of Bangladesh, and was found positive by an rK39 rapid immunochromatographic test (InBios International Inc., Seattle, Washington, USA). The period since the lesion first appeared was estimated to be three months, when he was working in the Kingdom of Saudi Arabia (KSA), a country known to be endemic for CL. Physical examination revealed one central depigmented ulcerated region surrounded by several hyperkeratotic, plaque like, sharply demarcated, painless papulonodular lesions (Fig. 1a) on his distal posteromedial aspect of the left forearm. Based on case history and clinical examination, a provisional diagnosis of CL was made and lesional biopsy was obtained for parasitological confirmation. Maintaining aseptic conditions, a $\sim 3.0 \mathrm{~mm}$ in diameter skin snip was collected with a scalpel from the nodular lesions followed by direct microscopy of the Giemsa-stained thin biopsy smear, which revealed large macrophages containing abundant intracellular and extracellular amastigotes $(3+$ parasitemia grade: 1-10 parasites/microscopic field). One additional snip of a nodular lesion as well as pictures of the lesions were collected following the patient's consent. The patient received a combination therapy of liposomal amphotericin B (AmBisome) at a dose of $20 \mathrm{mg} / \mathrm{kg}$ body-weight in four equally divided doses for four days.

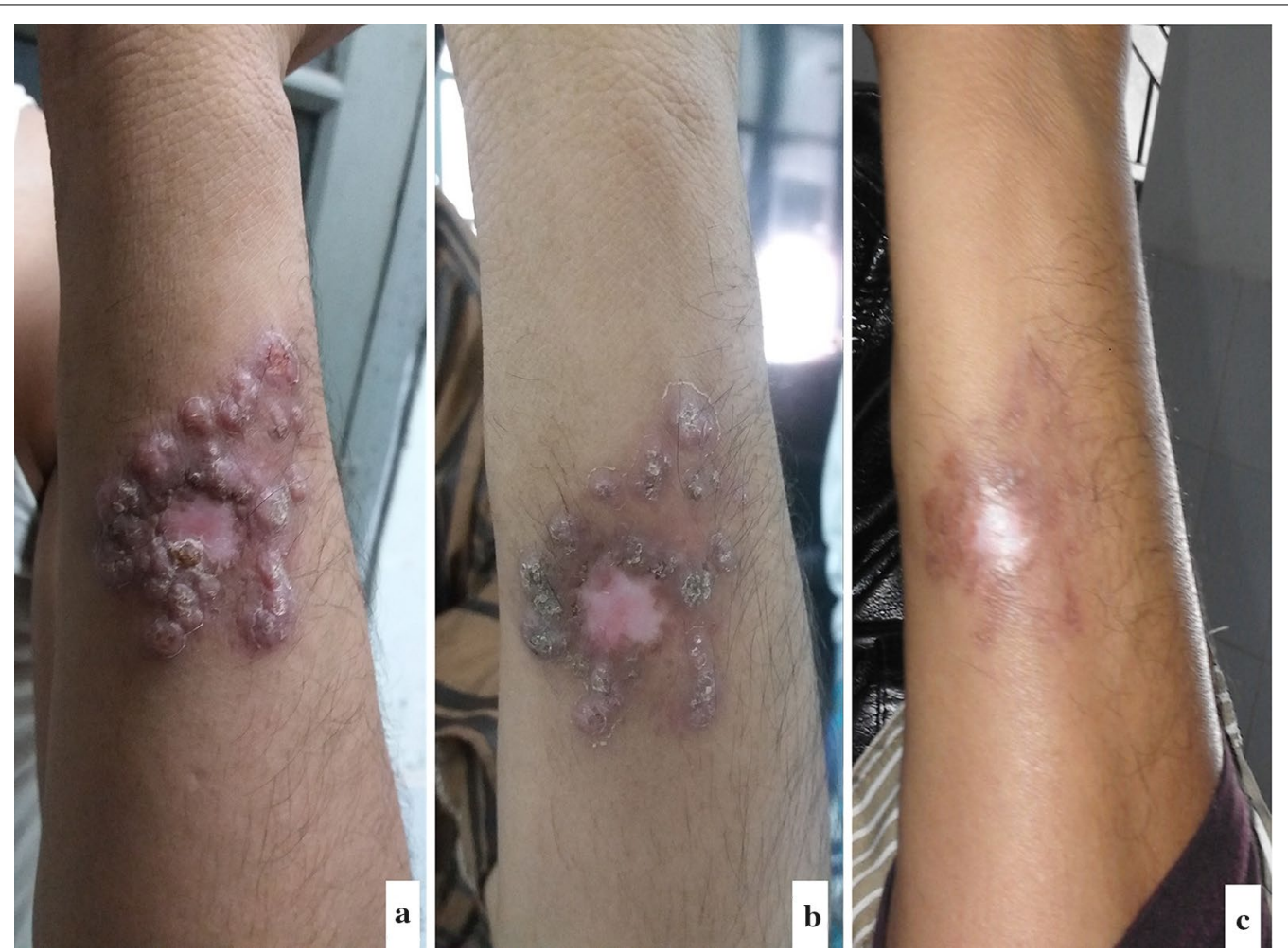

Fig. 1 Ulcerative lesion surrounded by nodules on the lower left arm of the patient, October 2017 to January 2018, SKKRC hospital, Mymensingh before treatment (a), five days after treatment with 4 doses of AmBisome (b) and twelve weeks after treatment with Miltefosine (c) 
This was followed by an oral Miltefosine capsule for 12 weeks at a dose of $100 \mathrm{mg} /$ day. The combination therapy resulted in a remarkable improvement, demonstrated by the dried up nodular crusts after five days (Fig. 1b), and disappearance of nodules leaving atrophic scars with hypopigmented spots in the middle after 12 weeks (Fig. 1c). No major side-effect was reported during the follow-up visits and the patient did not return with any relapse symptoms.

\section{Parasite culture and DNA extraction}

The additional skin snip $(\sim 3.0 \mathrm{~mm}$ in diameter $)$ collected from a nodular lesion was inoculated into RPMI1640 culture medium with $10 \%$ FBS supplemented with penicillin-streptomycin. Two volumes each of stationary phase culture promastigotes were inactivated, stored in buffer AL (Qiagen) at a 1:1 ratio and sent to the Emerging Infections and Parasitology laboratory of International Center for Diarrheal Disease Research (Dhaka, Bangladesh). DNA was extracted by using a QIAmp Blood DNA Mini Kit (Qiagen).

\section{Real-time PCR and sequencing}

A TaqMan probe based real-time (RT)-PCR assay, which targets the conserved region of Leishmania REPL repeats (L42486.1) of $L$. donovani complex, was carried out [5]. A threshold cycle (Cq) of 40- in a 45-cycle assay was considered positive. For species identification by sequencing, amplicons of the internal transcribed spacer region 2 (ITS2) were generated by PCR [6]. The amplicons were purified and prepared for Sanger sequencing by Microsynth Seqlab (Goettingen, Germany). Nucleotide BLAST search (NCBI) was used to estimate pairwise similarity of the tested sequence with reference Leishmania spp. genomes. A Tamura-Nei genetic distance model and neighbour-joining phylogenetic tree method for the derived ITS2 sequence was constructed together with sequences for Leishmania spp. with GENEIOUS v.9.1.6 (Biomatters Ltd., Auckland, New Zealand) using the incorporated tree builder at default settings.

\section{Results}

The RT-PCR assay did not result in a positive detection of L. donovani DNA in the culture sample. For identification of Leishmania species, therefore, the PCR amplified 400bp segment of ITS2 spacer was sequenced. The obtained sequence (Leish 17-832), which was assigned for species identification using nucleotide BLAST search (NCBI), showed pairwise similarity of $99 \%$ to L. major reference genome (GenBank: NC_007268) with a query cover of $100 \%$. In contrast, for the closest reference genome strain of L. infantum (GenBank: NW_004057905.1), the pairwise identity and query cover was $88 \%$ and $90 \%$, respectively (Table 1). The phylogenetic tree constructed for the obtained ITS2 sequence showed that $L$. major, originating possibly from Iran, shares a common ancestral node with the test sequence in a single branch (Fig. 2). The obtained nucleotide sequence was submitted to the GenBank database under the accession no. MK034756.

\section{Discussion}

In Bangladesh, VL and PKDL are prevalent in endemic areas, whereas CL, a localized manifestation of nodular or popular lesion with ulceration, is not regarded endemic. Because the presentation of CL mimics common diseases like tuberculosis, anthrax and fungal infections [3], it may cause a diagnostic dilemma, especially in non-endemic regions, which can lead to inappropriate clinical management. In this report, national guidelines were followed to define the clinical CL suspect, and diagnosis was confirmed parasitologically [7]. Since the patient was rK39 ICT positive and had previously visited a VL endemic area, RT-PCR assay positivity would have suggested a chance of mixed infection [8] with $L$. donovani. On the other hand, although rK39 ICT is a specific

Table 1 Results of NCBI online nucleotide BLAST search of the newly generated sequence (Leish 17-832) using the NCBI Genomic Reference Sequences Database

\begin{tabular}{|c|c|c|c|c|}
\hline Hit & GenBank ID & Pairwise identity (\%) & Query cover (\%) & e-value \\
\hline $\begin{array}{l}\text { Leishmania major strain Friedlin complete genome, } \\
\text { chromosome } 27\end{array}$ & NC_007268.2 & 99 & 100 & 0.0 \\
\hline $\begin{array}{l}\text { Leishmania infantum JPCM5 WGS CACT00000000 data, } \\
\text { contig 37, whole genome shotgun sequence }\end{array}$ & NW_004057905.1 & 88 & 90 & $7 e-105$ \\
\hline $\begin{array}{l}\text { Leishmania donovani BPK282A1 complete genome, } \\
\text { chromosome } 27\end{array}$ & NC_018254.1 & 88 & 90 & $1 e-102$ \\
\hline $\begin{array}{l}\text { Leishmania mexicana MHOM/GT/2001/U1103 WGS } \\
\text { CADB00000000 data, contig 148, whole genome } \\
\text { shotgun sequence }\end{array}$ & NW_003946471.1 & 87 & 87 & $7 e-95$ \\
\hline
\end{tabular}




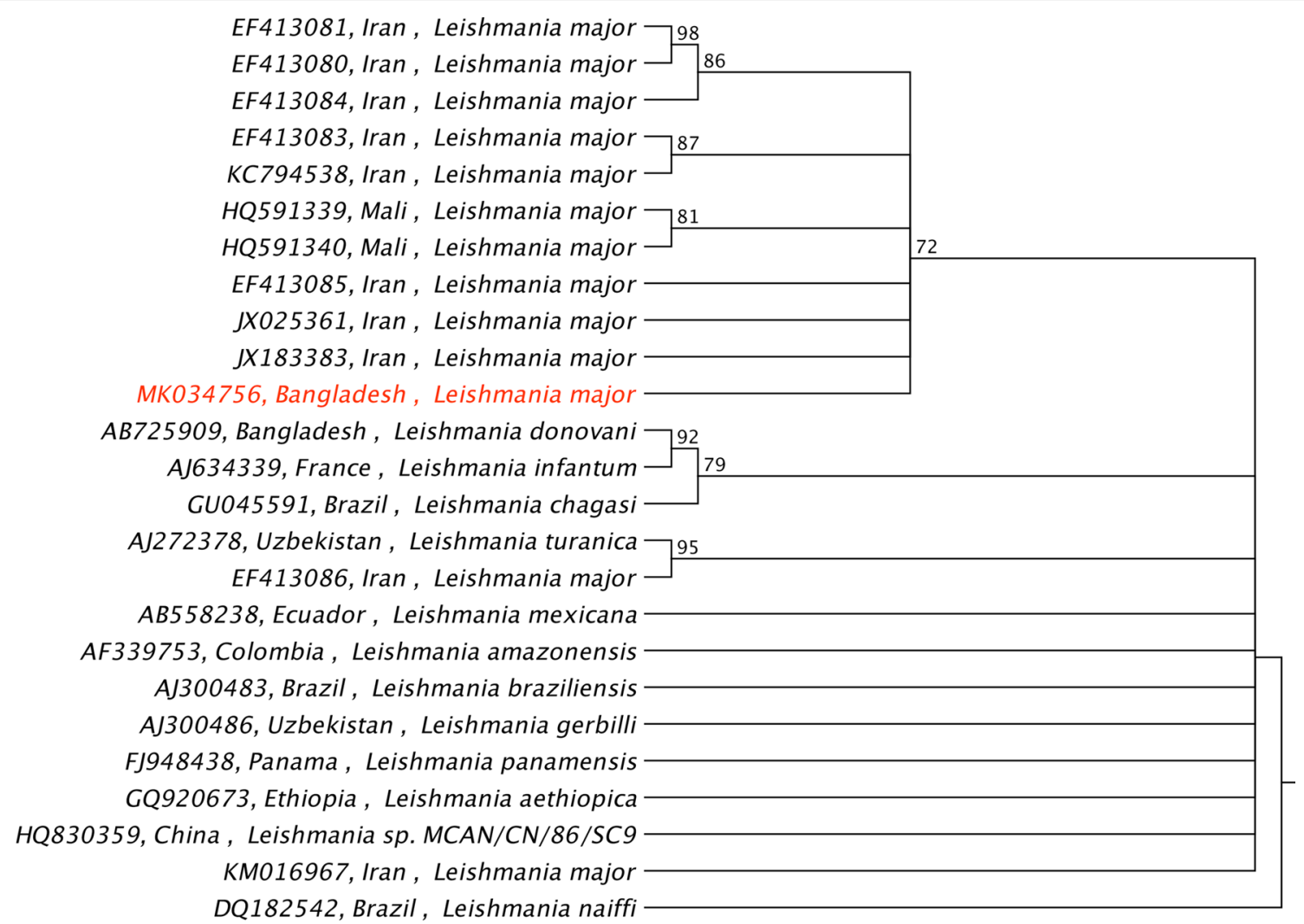

Fig. 2 Phylogenetic relationships of Leishmania species showing the position of the present sample (red) of Leishmania major

antibody marker test for active VL detection, its crossreactivity with sera from $\mathrm{CL}$ patients is also evident to some extent. Hence, the rK39 ICT test positivity could be associated either with an already healed infection with species that causes VL, species-specific cross-reactivity against CL-causing parasites [9-11], greater duration and severity of cutaneous infection [12] or region-specific phylogenetic proximity among species [13]. Finally, sequencing analysis of a species discriminatory segment of the ITS2 spacer [6] revealed that the obtained sequence (GenBank: MK034756) had almost 100\% similarity with the reference genome sequence of $L$. major for absolute query cover (Table 1). The phylogenetic tree indicates that the test sequence shares common ancestry with L. major strains that originated in Iran (Fig. 2). This is consistent with case travel history, as L. major and L. tropica are the main dermotropic species in the CL endemic regions of countries of the Middle East including KSA. Phlebotomus papatasi (vector of L. major) and $P$. sergenti (vector of $L$. tropica) are the proven vectors of the parasite in this region [2]. In Bangladesh, however, $P$. argentipes is the only known vector of $L$. donovani. Although a possible variant of $L$. donovani has also been prevalently causing CL in neighbouring countries,
India and Sri Lanka $[14,15]$, no such evidence is found in Bangladesh so far, and the other case reports of CL also indicated that the disease was imported from middleeastern regions $[16,17]$. Thus, CL can still be regarded as only an imported disease in Bangladesh.

Imported leishmaniasis has been becoming a globally emerging infectious disease in returned travellers; a 24-year analysis showed that more than $80 \%$ of those cases concerned CL [18]. Assessment of the risk of contracting CL, especially by Bangladeshi travellers, should be given careful consideration because the middle-eastern countries including KSA comprise one of the largest stocks of Bangladeshi migrants and temporary-migrant workers (>3.0 million) [19]. Due to the self-healing nature of CL, many of them may go underreported upon return, and be asymptomatic or subclinical. Subsequently, the chances of genetic exchange between parasites can be relevant within the context, because Leishmania is capable of cross-species and intra-clonal mating, which may increase parasite fitness [20]. Moreover, P. argentipes is not competent to only $L$. donovani, but rather permissive to other pathogenic species including L. major [21]. Natural adaptation of a new Leishmania species to the endemic vector [22] or co-existence of species and/or 
genetic variants [15] in endemic zones are not unusual. More importantly, considering that co-endemic zones of $\mathrm{VL}$ and $\mathrm{CL}$ are emerging in neighbouring countries $[15,23]$, screening and examination of imported CL suspects will be crucial to estimate the occurrence rate, and address whether such atypical cases can potentially become a new challenge for the control initiatives against leishmaniasis in Bangladesh.

\section{Conclusions}

In Bangladesh, imported cutaneous leishmaniasis is becoming more apparent. The imported CL case reported herein is, to our knowledge, the first evidence of $L$. major-derived pathology occurring in a Bangladeshi citizen. Our investigation indicates that presence of atypical cases in VL endemic areas can represent a diagnostic challenge, especially with antibody-based tests specific for active VL detection. Molecular tests should be performed in the diagnosis of such atypical cases to avoid dilemma. Furthermore, such cases should not be left out of epidemiological concern.

\begin{abstract}
Abbreviations
BLAST: basic local alignment search tool; CL: cutaneous leishmaniasis; ITS2: internal transcribed spacer 2; KAEP: kala-azar elimination program; KSA: Kingdom of Saudi Arabia; MCL: muco-cutaneous leishmaniasis; PKDL: post kala-azar dermal leishmaniasis; rk39 ICT: recombinant k39 immunochromatographic test; RT-PCR: real time polymerase chain reaction; VL: visceral leishmaniasis.
\end{abstract}

\section{Acknowledgements}

The publication of this study has been sponsored by Georg-August-Universität Göttingen.

\section{Authors' contributions}

MAAK overviewed laboratory methods, analysed the results and drafted the manuscript. RC performed the real-time PCR assay. RN performed microscopy and maintained parasite culture. SH performed the sequencing study. PN and SM managed the clinical case and revised the manuscript. AAEW supervised sequencing, data analysis and revised the manuscript. DM designed and coordinated the study and critically reviewed the manuscript. All authors read and approved the final manuscript.

\section{Funding}

The publication fees was covered by the Open Access Publication Funds of the University of Goettingen, Germany.

\section{Availability of data and materials}

Not applicable.

\section{Ethics approval and consent to participate}

Written informed consent was obtained from the patient to take additional skin specimen and photographs of the affected area for use in research and publication without revealing identity.

\section{Consent for publication}

Patient agreed for the publication of the study.

\section{Competing interests}

The authors declare that they have no competing interests.

\section{Author details}

${ }^{1}$ Nutrition and Clinical Services Division, International Centre for Diarrhoeal Disease Research, Bangladesh, Dhaka, Bangladesh. ${ }^{2}$ Division of Microbiology and Animal Hygiene, Georg-August-Universität Göttingen, Göttingen, Germany. ${ }^{3}$ Surya Kanta Kala-azar Research Center, Mymensingh, Bangladesh.

Received: 21 June 2019 Accepted: 25 October 2019

Published online: 07 November 2019

References

1. Hirve S, Kroeger A, Matlashewski G, Mondal D, Banjara MR, Das P, et al. Towards elimination of visceral leishmaniasis in the Indian subcontinenttranslating research to practice to public health. PLoS Negl Trop Dis. 2017;11:e0005889.

2. Maroli M, Feliciangeli MD, Bichaud L, Charrel RN, Gradoni L. Phlebotomine sandflies and the spreading of leishmaniases and other diseases of public health concern. Med Vet Entomol. 2013;27:123-47.

3. Goto $\mathrm{H}$, Lindoso JA. Current diagnosis and treatment of cutaneous and mucocutaneous leishmaniasis. Expert Rev Anti Infect Ther. 2010;8:419-33.

4. Vanaerschot M, Dumetz F, Roy S, Ponte-Sucre A, Arevalo J, Dujardin JC. Treatment failure in leishmaniasis: drug-resistance or another (epi-) phenotype? Expert Rev Anti Infect Ther. 2014;12:937-46.

5. Vallur AC, Duthie MS, Reinhart C, Tutterrow Y, Hamano S, Bhaskar KR, et al. Biomarkers for intracellular pathogens: establishing tools as vaccine and therapeutic endpoints for visceral leishmaniasis. Clin Microbiol Infect. 2014;20:0374-83.

6. de Almeida ME, Steurer FJ, Koru O, Herwaldt BL, Pieniazek NJ, da Silva AJ. Identification of Leishmania spp. by molecular amplification and DNA sequencing analysis of a fragment of rRNA internal transcribed spacer 2. J Clin Microbiol. 2011;49:3143-9.

7. Directorate General of Health Services, Ministry of Health and Family Welfare, Govt. of People Republic of Bangladesh. Diagnosis of kala-azar and PKDL. In: National guideline for kala-azar case management 2016. 3rd ed; 2016. p. 18-27. http://kalacorebd.com/wp-content/uploads/2016/04/ Kala-azar-Case-Managment-Guideline-2016_Latest-Draft.pdf. Accessed 21 Feb 2019.

8. Babiker AM, Ravagnan S, Fusaro A, Hassan MM, Bakheit SM, Mukhtar MM, et al. Concomitant infection with Leishmania donovani and L. major in single ulcers of cutaneous leishmaniasis patients from Sudan. J Trop Med. 2014;2014:170859.

9. Molinet FJ, Ampuero JS, Costa RD, Noronha EF, Romero GA. Specificity of the rapid rK39 antigen-based immunochromatographic test Kalazar Detect(r) in patients with cutaneous leishmaniasis in Brazil. Mem Inst Oswaldo Cruz. 2013;108:293-6.

10. Kumar NP, Srinivasan R, Anish TS, Nandakumar G, Jambulingam P. Cutaneous leishmaniasis caused by Leishmania donovani in the tribal population of the Agasthyamala Biosphere Reserve forest, Western Ghats, Kerala, India. J Med Microbiol. 2015;64:157-63.

11. Saghrouni F, Gaïed-Meksi S, Fathallah A, Amri F, Ach H, Guizani I, et al. Immunochromatographic rK39 strip test in the serodiagnosis of visceral leishmaniasis in Tunisia. Trans R Soc Trop Med Hyg. 2009;103:1273-8.

12. Hartzell JD, Aronson NE, Weina PJ, Howard RS, Yadava A, Wortmann GW. Positive rK39 serologic assay results in US servicemen with cutaneous leishmaniasis. Am J Trop Med Hyg. 2008;79:843-6.

13. Mahdy MA, Al-Mekhlafi HM, Al-Mekhlafi AM, Lim YA, Bin Shuaib NO, Azazy AA, et al. Molecular characterization of Leishmania species isolated from cutaneous leishmaniasis in Yemen. PLoS ONE. 2010;5:e12879.

14. Siriwardana HV, Noyes HA, Beeching NJ, Chance ML, Karunaweera ND, Bates PA. Leishmania donovani and cutaneous leishmaniasis, Sri Lanka. Emerg Infect Dis. 2007;13:476-8.

15. Thakur L, Singh KK, Shanker V, Negi A, Jain A, Matlashewski G, et al. Atypical leishmaniasis: a global perspective with emphasis on the Indian subcontinent. PLoS Negl Trop Dis. 2018;12:e0006659.

16. Rahman H, Razzak MA, Chanda BC, Bhaskar KR, Mondal D. Cutaneous leishmaniasis in an immigrant Saudi worker: a case report. J Health Popul Nutr. 2014;32:372-6.

17. Basher A, Nath P, Dey T, Sayeed AA, Faiz MA, Chowdhury FR. Cutaneous leishmaniasis in immigrant workers returning to Bangladesh-an emerging problem. Travel Med Infect Dis. 2017;19:62-3. 
18. Pavli A, Maltezou HC. Leishmaniasis, an emerging infection in travelers. Int J Infect Dis. 2010;14:e1032-9.

19. Benjamin E, Bishawjit M. International migration from Bangladesh. In: Country profile migration: data-history-politics (data source: Trends in international migrant stock: the 2013 revision-migrants by destination and origin, UN database); 2015. https://www.bpb.de/gesellschaft/migra tion/laenderprofile/216104/international-migration-from-bangladesh. Accessed 21 Feb 2019.

20. Romano A, Inbar E, Debrabant A, Charmoy M, Lawyer P, Ribeiro-Gomes $F$, et al. Cross-species genetic exchange between visceral and cutaneous strains of Leishmania in the sand fly vector. Proc Natl Acad Sci USA. 2014;111:16808-13.

21. Svarovska A, Ant TH, Seblova V, Jecna L, Beverley SM, Volf P. Leishmania major glycosylation mutants require phosphoglycans (lpg2-) but not lipophosphoglycan (lpg1-) for survival in permissive sand fly vectors. PLoS Negl Trop Dis. 2010;4:e580.

22. Mauricio IL, Stothard JR, Miles MA. The strange case of Leishmania chagasi. Parasitol Today. 2000;16:188-9.

23. Siriwardana HVYD, Karunanayake P, Goonerathne L, Karunaweera ND. Emergence of visceral leishmaniasis in Sri Lanka: a newly established health threat. Pathog Glob Health. 2017;111:317-26.

\section{Publisher's Note}

Springer Nature remains neutral with regard to jurisdictional claims in published maps and institutional affiliations.
Ready to submit your research? Choose BMC and benefit from:

- fast, convenient online submission

- thorough peer review by experienced researchers in your field

- rapid publication on acceptance

- support for research data, including large and complex data types

- gold Open Access which fosters wider collaboration and increased citations

- maximum visibility for your research: over $100 \mathrm{M}$ website views per year

At BMC, research is always in progress.

Learn more biomedcentral.com/submissions 